\title{
Open-Angle Glaucoma and Paraoptic Cyst: First Description of a Series of 11 Patients
}

\author{
A. Bertrand, C. Vignal, F. Lafitte, P. Koskas, O. Bergès, and F. Héran
}

\begin{abstract}
SUMMARY: We report 11 patients who were referred to our institution for severe open-angle glaucoma who had a paraoptic cyst on MR imaging. All cysts were extraoptic and retrolaminar; most were deforming the adjacent optic nerve. Cysts had a high signal on T2 and FLAIR sequences, and a variable signal on $\mathrm{Tl}$ and variable echogenicity, suggesting different proteinaceous content. Arterial vascularization of the optic nerve was normal. Cyst volumes were inversely correlated with the severity of glaucoma on the same eye $(P<.01-.05$, Spearman correlation coefficient). We hypothesized that such cysts may reflect a valve mechanism, which would allow preservation of the translamina cribrosa pressure and thus could preserve visual function. The rarity of this association, together with the frequent mass effect of the cyst on the optic nerve, stresses the necessity of long-term follow-up in these patients.
\end{abstract}

ABBREVIATIONS: $M D=$ mean deviation; OCT = optical coherence tomography; PSD = pattern standard deviation; US = ultrasound

$\mathbf{P}$ rimary open-angle glaucoma, a chronic optic neuropathy characterized by a progressive excavation of the optic nerve and an associated loss of visual field sensitivity, affects approximately $2 \%$ of the population older than 40 years of age and represents a leading cause of irreversible blindness worldwide. ${ }^{1}$ The pathogenic mechanisms of open-angle glaucoma are still a matter of debate. ${ }^{2-4}$ Until the 1970s, primary open-angle glaucoma was considered the consequence of a single cause, the increase in intraocular pressure; but it is now recognized that approximately one-third of patients with glaucomatous neuropathy have normal intraocular pressure. ${ }^{5}$ Recently, some authors have proposed that open-angle glaucoma may be driven not by the increased intraocular pressure but by an increased translamina cribrosa pressure gradient (ie, an increased difference of pressure between the intraocular compartment and the retrobulbar compartment [CSF compartment]). ${ }^{6}$ This hypothesis is supported by recent experimental and clinical data showing an association between the oc-

Received March 5, 2014; accepted after revision September 28.

From the Departments of Diagnostic Neuroradiology (A.B., F.L., P.K., O.B., F.H.) and Ophthalmology (C.V.), Fondation Ophtalmologique Adolphe de Rothschild, Paris, France; and Department of Diagnostic and Functional Neuroradiology (A.B.), PitiéSalpétrière Hospital, Paris, France.

Paper previously presented at: Annual Meeting of the American Society of Neuroradiology, April 21-26, 2012; New York, New York.

Please address correspondence to Françoise Héran, MD, Department of Neuroradiology, Fondation Adolphe de Rothschild, 25 rue Manin, 75019 Paris, France; e-mail: fheran@fo-rothschild.fr

三 Indicates article with supplemental on-line table.

http://dx.doi.org/10.3174/ajnr.A4194 currence of normal-pressure glaucoma and decreased CSF pressure. $^{6-9}$

In this work, we present 11 patients referred to our institution for severe open-angle glaucoma in whom MR imaging demonstrated the presence of a retrolaminar paraoptic cyst at the head of the optic nerve. We collected the ophthalmologic reports of these patients, together with the results of MR imaging of the optic nerves and, when available, high-resolution MR imaging and Doppler ultrasound (US) examination of the optic nerves. We describe the morphologic characteristics of these cysts and discuss associated morphologic, vascular, and/or functional signs of optic neuropathy to determine their pathophysiologic significance.

\section{MATERIALS AND METHODS \\ Case Series}

Patients. We retrospectively collected the cases of 11 patients who were referred to our institution for severe open-angle glaucoma who were diagnosed on MR imaging of the optic nerves as having a paraoptic cyst from 2005 to 2011 . We estimate that during this period, approximately 200 patients underwent brain MR imaging for the same indication. This retrospective study was approved by our institutional review board. Explicit informed consent was waived according to French legislation because all imaging and clinical data were generated during routine clinical work-up and were retrospectively extracted for this study.

Ophthalmologic Examinations. For 9 patients (10 cysts), we collected the results of visual field tests (24-2) on both eyes; the 2 other patients had no records in our institution. Defects on visual 
field tests were retrospectively analyzed qualitatively by an ophthalmologist (C.V.) with 20 years' experience and quantitatively by using the values of mean deviation (MD) and pattern standard deviation (PSD). MD is the mean difference in decibels between the patient's measured field of vision and a normal reference field model. PSD is the sum of the deficits in decibels on the patient's measured field of vision; an impairment of the visual field corresponds to decreased MD and an increased PSD. Seven patients (8 cysts) also underwent an optical coherence tomography (OCT) on both eyes. OCT is a noninvasive optical imaging technique that allows the measurement of the nerve fiber thickness at the head of the optic nerve. ${ }^{10}$

MR Imaging Examinations. All patients had a standard MR imaging examination of the optic nerves. Standard MR imaging was performed on a 1.5T Gyroscan ACS-NT scanner (Philips Healthcare, Best, the Netherlands) with an 8-channel head coil and always included a 2-mm-thick coronal T2-weighted sequence perpendicular to the optic nerves. Seven patients also had highresolution MR imaging of the optic nerves. High-resolution MR imaging was performed on the same magnet by using a surface coil placed on the eyes and comprised a $3 \mathrm{D}$ driven equilibrium sequence acquired in the axial plane $(\mathrm{TR} / \mathrm{TE}=1500 / 250 \mathrm{~ms}$, voxel size $\left.=0.51 \times 0.65 \times 1.6 \mathrm{~mm}^{3}\right)$, a coronal T1-weighted sequence $(\mathrm{TR} / \mathrm{TE}=500 / 20 \mathrm{~ms}$, voxel size $=0.69 \times 0.87 \times 2$ $\left.\mathrm{mm}^{3}\right)$, a coronal T2-weighted sequence $(\mathrm{TR} / \mathrm{TE}=2975 / 100 \mathrm{~ms}$, voxel size $=0.59 \times 0.81 \times 2 \mathrm{~mm}^{3}$ ), and a coronal turbo-FLAIR sequence $(\mathrm{TR} / \mathrm{TE} /$ inversion recovery time $=11,000 / 155 / 2800$ $\mathrm{ms}$, TSE factor $=50$, voxel size $=0.59 \times 0.75 \times 2 \mathrm{~mm}^{3}$ ). Cyst volumes were measured on coronal $2 \mathrm{D}-\mathrm{T} 2$ in all 11 patients and on $3 \mathrm{D}$ driven equilibrium in the 7 patients who underwent highresolution MR imaging. Volume measurement was done by image-by-image manual delineation, by using OsiriX Imaging Software, Version 5.5.2 (http://www.osirix-viewer.com).

US and Doppler Examinations. US and Doppler examination of the optic nerves were available in 9 patients (10 cysts). Doppler examination included the bilateral measurement of the resistance index in the ophthalmic, central retinal, nasal short posterior ciliary, and temporal short posterior ciliary arteries of both eyes. For every patient, the radiologist performing the Doppler US examination (O.B., F.L., or P.K.) was guided by the MR images of the patient's cyst.

Statistical Analysis. Data gathering and analysis were performed by A.B. Given our small sample size, only nonparametric tests were used in this study. MD, PSD, and OCT values were compared between the side of the cyst and the opposite side in the 8 patients with unilateral cysts; and available visual field tests and OCT, by using the Wilcoxon matched pair test. Arterial resistance indices were compared between the side of the cyst and the opposite side in the 7 patients who had a unilateral cyst and available US imaging, by using the Wilcoxon matched pair test. Correlation between the size of the cysts on the one hand and the indicators of glaucoma severity on the side of the cyst on the other hand (MD and PSD on visual field, thickness of the nerve fiber layer on OCT) was assessed by using the Spearman correlation coefficient.

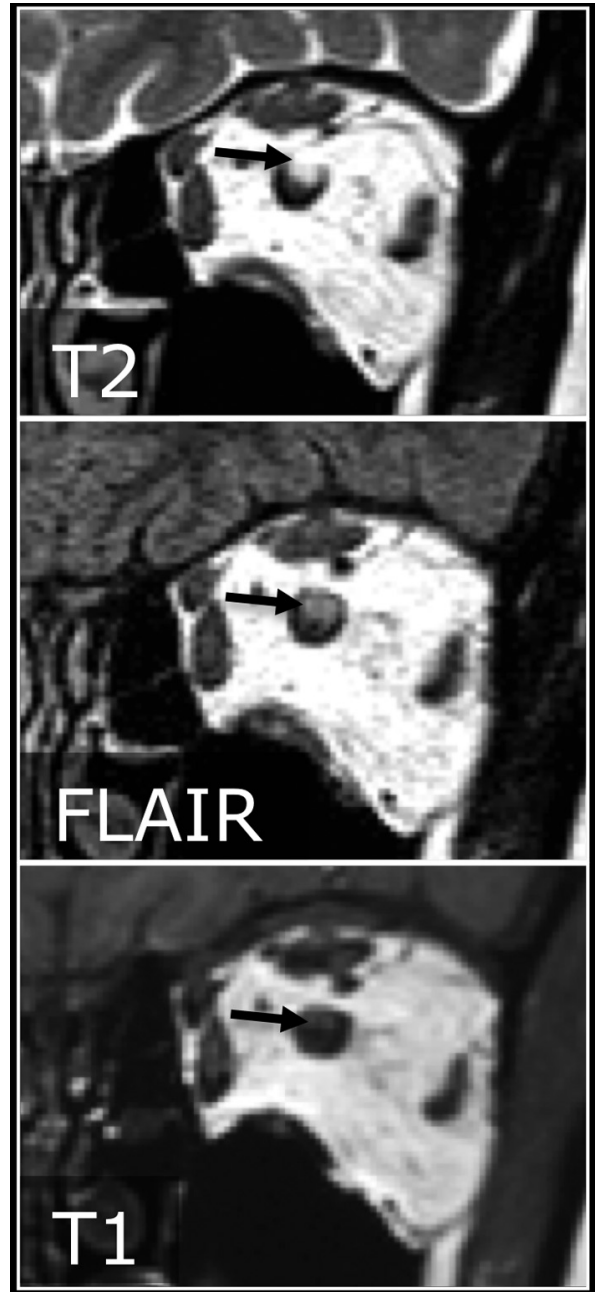

FIG 1. High-resolution MR images of a paraoptic cyst. The cyst is superolateral, causing a mass effect on the optic nerve, which is displaced inward and inferiorly. The content of the cyst appears hyperintense on T2WI and moderately hyperintense on FLAIR and TTWI.

\section{RESULTS}

\section{Patient Characteristics}

Patients were 4 men and 7 women, $48-82$ years of age (median, 70 years). All patients were previously diagnosed with open-angle glaucoma; 4 had normal-pressure glaucoma (On-line Table).

\section{Cyst Morphology}

All cysts were located at the level of the optic nerve head in the retrolaminar area. In 5 patients, the cyst was located on the right optic nerve; in 5 patients, on the left optic nerve; and 1 patient had bilateral cysts. All cysts were located outside the optic nerve, and all except 2 were associated with a mass effect on the adjacent optic nerve. Cysts were located inferior $(n=1)$, inferolateral $(n=3)$, superolateral $(n=2)$, or superior $(n=6)$ to the optic nerve (On-line Table). All cysts were hyperintense on T2WI. On highresolution MR images, the signal of the cysts was always high on T2WI, always high on FLAIR-weighted imaging, and variable on T1WI (hyperintense in 1 case, hypointense in 1 case, and of mixed intensities in the remaining cases) (Fig 1). Cyst volume ranged from 4 to $54 \mathrm{~mm}^{3}$ on coronal T2 sequences (median, 18) and from 10 to $58 \mathrm{~mm}^{3}$ on $3 \mathrm{D}$-CISS sequences (median, 21). Among the 8 
patients who underwent US imaging, 5 cysts were visible, either as a simple enlargement of the optic nerve $(n=3)$ or as an iso- to slightly hyperechoic formation, with a location and shape similar to those observed on MR imaging $(n=2)$ (Fig 2$)$.

\section{Doppler US}

No hemodynamic perturbation of the blood supply to the optic nerve head was observed on Doppler examination. There was no statistically significant difference between the resistance indices measured on the side of the cyst and those on the opposite side, for the ophthalmic, central retinal, nasal short posterior ciliary, and temporal short posterior ciliary arteries (Table).

\section{Correlation between Cyst Characteristics and Optic Neuropathy}

Absence of Correlation between Cyst Location and Topography of Optic Neuropathy. One ophthalmologist (C.V.) qualitatively assessed the correlation between the location of the cyst on MR images and the location of visual field defects on the retina. This correlation could only be qualitative because the examiner needed to take into account the uneven spatial distribution of optic fibers from the head of the optic nerve toward the retina. In 2 cases, there was complete correlation between the location of the cyst and the visual field defects; in 5 cases, this correlation was only

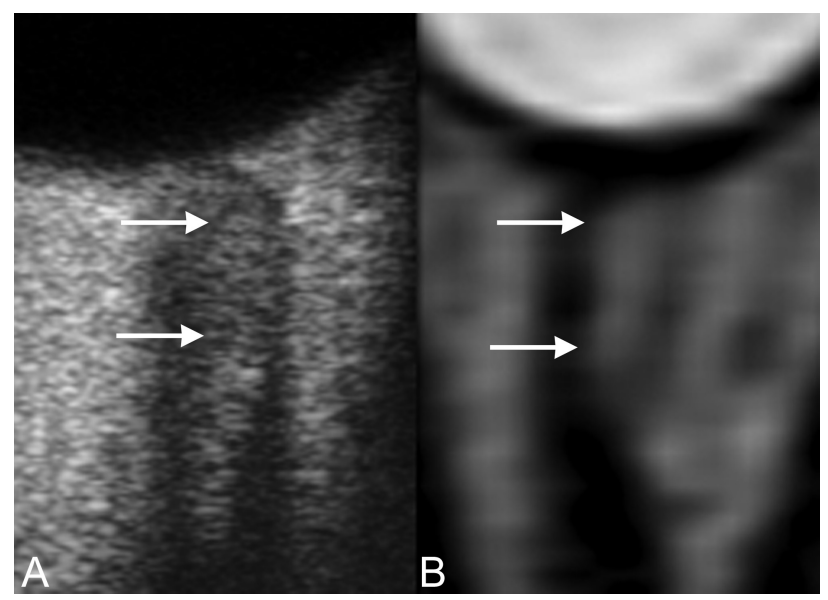

FIG 2. Axial image on US $(A)$ shows a lateral paraoptic cyst, welllimited and isoechogenic. Axial-reformatted 3D-CISS in the same patient $(B)$ shows a similar image of an oval hyperintense cyst. partial; and in the last 3 cases, there was no correlation between the location of the cyst and the visual field defects (On-line Table). Moreover, there was no statistical difference in MD and PSD values in the eye with the cyst and the eye without a cyst (paired $t$ test, $P=.38$ and .47 , respectively; data not shown). We also quantitatively assessed the correlation between the location of the cyst and the severity of nerve fiber loss on OCT. This correlation was unbiased and quantitative because the OCT technique gives a direct estimation of the number of fibers at the head of the optic nerve, where the cysts are located. We calculated the percentage of fiber loss on OCT in the 4 quadrants (nasal, temporal, superior, and inferior). For 4 cysts, the location of the cyst matched the quadrant of greatest fiber loss; in the 4 other cysts, the location of the cyst was in another quadrant. There was no statistical difference between fiber thickness in the quadrant where the cyst was located and in the same quadrant on the contralateral eye (Wilcoxon signed rank test, $P=.44$; data not shown).

Correlation between Cyst Size and Severity of the Optic Neuropathy. We quantitatively correlated the volume of the cysts and the severity of the glaucomatous neuropathy in the 6 patients (7 cysts) who underwent high-resolution MR imaging and for whom ophthalmologic reports were available. There was no correlation between the volume of the cyst in 3D-CISS and the thickness of the optic nerve fibers on OCT (Spearman correlation coefficient: $r=-0.25, P=.59$; Fig $3 A$ ). However, we noted a statistically significant correlation between the volume of the cyst in 3D-CISS and the severity of visual impairment in the same eye, estimated by the MD and PSD: The more voluminous the cyst was, the less impaired the visual field was (Fig $3 B,-C$ ). As estimated by linear regression, we would expect a PSD $=0$ for a cyst measuring $82 \mathrm{~mm}^{3}$ on 3D driven equilibrium; similarly, we would expect an $\mathrm{MD}=0$ for a cyst measuring $67 \mathrm{~mm}^{3}$ on $3 \mathrm{D}$ driven equilibrium. No significant correlation was observed between the volume of the cyst estimated on 2D-T2 and the MD or the PSD (On-line Table).

\section{DISCUSSION}

MR imaging examination of the orbit is not typically integrated into the systemic work-up in patients with open-angle glaucoma, but it is recommended when visual deficits are severe and/or rapidly increasing, to rule out a compressive tumor or other structural pathology of the anterior visual pathways. ${ }^{5}$ We report the association between open-angle glaucoma and paraoptic cysts.

\section{Comparison of arterial resistance index values between the side of the cyst and the contralateral eye (Wilcoxon matched pair test)}

\begin{tabular}{|c|c|c|c|c|c|c|c|c|}
\hline \multirow[b]{2}{*}{ Case No. } & \multicolumn{2}{|c|}{ Central Retinal Artery } & \multicolumn{2}{|c|}{ Temporal Short Ciliary Artery } & \multicolumn{2}{|c|}{ Nasal Short Ciliary Artery } & \multicolumn{2}{|c|}{ Ophthalmic Artery } \\
\hline & Cyst Side & Contra. & Cyst Side & Contra. & Cyst Side & Contra. & Cyst Side & Contra. \\
\hline 1 & 0.59 & 0.67 & 0.65 & 0.58 & 0.52 & 0.48 & 0.76 & 0.75 \\
\hline 3 & 0.59 & 0.6 & 0.53 & 0.56 & 0.61 & 0.5 & 0.66 & 0.68 \\
\hline $4 a$ & 0.62 & NA & 0.64 & NA & 0.65 & NA & 0.62 & NA \\
\hline $4 b$ & 0.63 & NA & 0.56 & NA & 0.5 & NA & 0.67 & NA \\
\hline 6 & 0.64 & 0.71 & NA & NA & 0.59 & 0.63 & 0.66 & 0.74 \\
\hline 8 & 0.54 & 0.69 & 0.59 & 0.52 & 0.55 & 0.56 & 0.66 & 0.66 \\
\hline 9 & 0.64 & 0.61 & 0.64 & 0.6 & 0.61 & 0.61 & 0.77 & 0.82 \\
\hline 10 & 0.77 & 0.77 & 0.69 & 0.77 & 0.74 & 0.75 & 0.75 & 0.73 \\
\hline 11 & 0.6 & 0.58 & 0.48 & 0.44 & 0.48 & 0.53 & 0.7 & 0.7 \\
\hline Mean (SD) & $0.62(0.06)$ & $0.66(0.3)$ & $0.6(0.21)$ & $0.58(0.3)$ & $0.58(0.08)$ & $0.58(0.27)$ & $0.69(0.05)$ & $0.73(0.32)$ \\
\hline Paired $t$ test & \multicolumn{2}{|c|}{$P=.18$} & \multicolumn{2}{|c|}{$P=.49$} & \multicolumn{2}{|c|}{$P=.79$} & \multicolumn{2}{|c|}{$P=.25$} \\
\hline
\end{tabular}

Note:-Contra. indicates contralateral. 


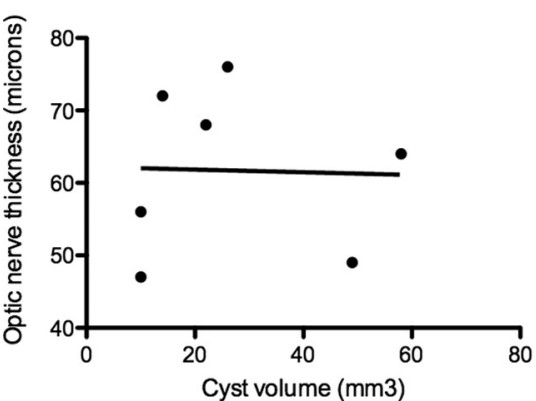

A

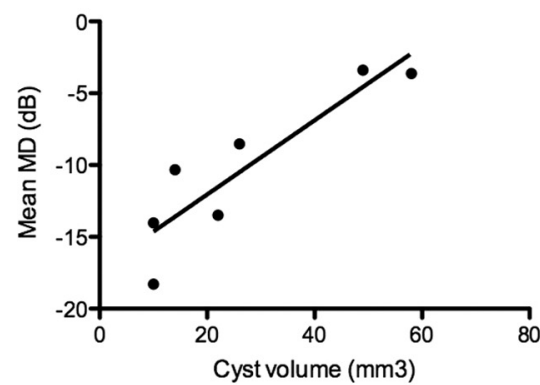

B $r=0,92 ; p<0,01$ (Spearman correlation coefficient)

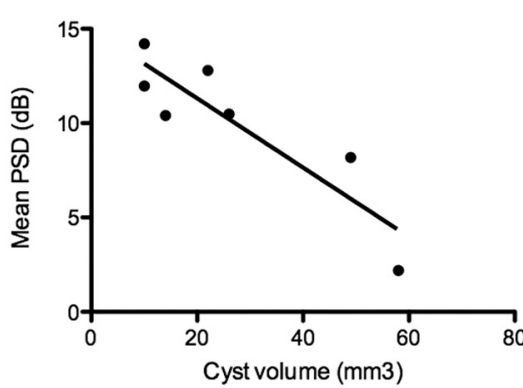

C $r=-0,79 ; p<0,05$ (Spearman correlation coefficient)

FIG 3. Correlation between cyst volumes on $3 D$-CISS and optic nerve fibers on OCT (A), MD (B), and PSD (C).

This association remains quite rare: We estimate that approximately 200 patients were referred to our institution for brain MR imaging because of severe open-angle glaucoma during a 6-year period; thus, we estimate the prevalence of these cyst to be at approximately $5 \%$ in patients with severe open-angle glaucoma. Other differential diagnoses of a paraoptic mass would include arachnoid cyst, coloboma, and pilocytic astrocytoma; however, the high signal of the cysts on FLAIR images was not in favor of a subarachnoid cyst, and the cyst location, distinct from both the optic nerve and the ocular globe, was not in favor of a tumor arising from the optic nerve, nor of a coloboma.

In our series of 11 patients, the presence of a cyst was not associated with a greater fiber loss in the head of the optic nerve, but it was associated with relatively preserved visual function. This goes against a mechanism of compression of the optic nerve by the cyst and does not suggest that surgical removal of the cyst would be of any benefit. Instead, it would suggest that for a given level of fiber loss, the cyst has some protective effect on visual function. It has been recently proposed that primary open-angle glaucoma could be driven not only by increased ocular pressure but, more generally, by an increase in translamina cribrosa pressure, either caused by elevated intraocular pressure or a diminished CSF pressure. In light of this pathogenic hypothesis, paraoptic cysts may represent a valve mechanism, by which a leakage of fluid from the ocular compartment to the optic nerve compartment would allow stabilization of the translamina cribrosa pressure and, consequently, relative preservation of visual function. However, this hypothesis remains speculative. The observed mass effect of the cyst on the optic nerve does not favor the idea that such cysts are purely protective. In addition, given the rarity of this association, our findings may not be generalized to all forms of open-angle glaucoma. It is also conceivable that distinct mechanisms underlie open-angle glaucoma with elevated pres- sure and open-angle glaucoma with normal pressure. In our small series, we did not observe different characteristics in these 2 groups, but this feature may simply be related to the small sample size. Consequently, long-term follow-up is warranted in patients with this MR imaging finding, to evaluate the long-term effects of these paraoptic cysts on visual function.

Disclosures: Catherine Vignal—UNRELATED: Consultancy: GenSight.

\section{REFERENCES}

1. Friedman DS, Wolfs RC, O'Colmain BJ, et al. Prevalence of openangle glaucoma among adults in the United States. Arch Ophthalmol 2004;122:532-38

2. Rieck J. The pathogenesis of glaucoma in the interplay with the immune system. Invest Ophthalmol Vis Sci 2013;54:2393-409

3. Mozaffarieh M, Flammer J. New insights in the pathogenesis and treatment of normal tension glaucoma. Curr Opin Pharmacol 2013;13:43-49

4. Venkataraman ST, Flanagan JG, Hudson C. Vascular reactivity of optic nerve head and retinal blood vessels in glaucoma: a review. Microcirculation 2010;17:568-81

5. Kwon $\mathrm{YH}$, Fingert JH, Kuehn MH, et al. Primary open-angle glaucoma. N Engl J Med 2009;360:1113-24

6. Ren $\mathrm{R}$, Zhang X, Wang N, et al. Cerebrospinal fluid pressure in ocular hypertension. Acta Ophthalmol 2011;89:e142-48

7. Ren R, Jonas JB, Tian G, et al. Cerebrospinal fluid pressure in glaucoma: a prospective study. Ophthalmology 2010;117:259-66

8. Berdahl JP, Allingham RR, Johnson DH. Cerebrospinal fluid pressure is decreased in primary open-angle glaucoma. Ophthalmology 2008;115:763-68

9. Ren R, Wang N, Zhang X, et al. Trans-lamina cribrosa pressure difference correlated with neuroretinal rim area in glaucoma. Graefes Arch Clin Exp Ophthalmol 2011;249:1057-63

10. Kotowski J, Wollstein G, Folio LS, et al. Clinical use of OCT in assessing glaucoma progression. Ophthalmic Surg Lasers Imaging 2011; 42(suppl):S6-14 